\title{
Regulatory T cells in cancer; can they be controlled?
}

\author{
"...the race toward 'Treg-centric' cancer treatment continues and the \\ answer to the topic question remains a half yes."
}

\begin{abstract}
Keywords: cancer $\bullet$ chemokines $\bullet$ function $\bullet$ immune checkpoint $\bullet$ immunotherapy - microenvironment $\bullet$ recruitment $\bullet$ regulatory T cell $\bullet$ TCR diversity $\bullet$ tumor-associated antigen
\end{abstract}

Natural regulatory $\mathrm{T}$ (Treg) cells, described as $\mathrm{CD} 4^{+} \mathrm{CD} 25^{+}$Foxp $3^{+}$cells have long been established as a subset of $\mathrm{T}$ lymphocytes critical for maintaining tolerance to selfconstituents [1]. Their suppressive activities however extend beyond self tolerance as they have been demonstrated to regulate various facets of immune responses against allergens, microbial and infectious agents, as well as tissue-derived alloantigens [1]. Mounting evidence also shows their increased presence in cancer settings, often coinciding with attenuated antitumor T-cell responses [2]. Although there is limited direct evidence to the antigen specificity of tumor-associated Treg cells, given that they develop in the thymus and seed the periphery primarily to curtail the activities of $T$ cells that may have escaped thymic negative selection, it stands to reason that they are specific for tumor antigens which invariably are often self protein, albeit in altered or aberrantly expressed forms. What prompts Treg-cell accumulation within tumor microenvironment? What cues are necessary, what exactly are they regulating, and how might some of these issues be tackled for therapeutic purposes? These are questions in the minds of many cancer biologists and clinicians, and the answers could pave way to new therapeutic modalities. Although Treg-cell biology is a vast subject, we will attempt here to provide a brief synopsis of these cells in cancer and whether they can be controlled to achieve therapeutic outcomes.

\section{Regulatory T cells in cancer: recruitment \& function}

Several animal tumor models and human cancers harbor higher frequencies of Treg cells than those generally seen in the peripheral blood or lymphoid compartments [3]. Their over-representation often overshadow the presence of $\mathrm{CD}^{+} \mathrm{T}$ cells, resulting in a low CD8:Treg ratio that has been associated with poor prognosis in some studies [4]. The increased presence of Treg cells within the tumor is likely a combination of recruitment from lymphoid compartments as well as de novo conversion of Foxp3-negative cells into Foxp3-expressing cells. A number of chemokines and receptors have been implicated in the recruitment of Treg cells into the tumor microenvironment [5]. Secreted by tumor cells and/or inflammatory myeloid cells, CCL5, CCL17, CCL22, CCL28, and CXCL9, 10 and 11 for instance are abundant in pancreatic, gastric, ovarian and breast cancers, creating a chemoattractant gradient that recruits $\mathrm{T}$ lymphocytes particularly Treg cells into the tumor [2,5]. Indeed, the receptors for these chemokines have been shown to be highly expressed on Treg cells, consistent with their preferential recruitment over conventional CD $4^{+} \mathrm{T}$ cells $([5,6])$. Other soluble factors and cytokines such as IL-10, TGF- $\beta$, Indoleamine 2,3-dioxygenase (IDO), may promote the conversion of conventional $\mathrm{CD}^{+}{ }^{+} \mathrm{T}$ cells to become induced or adaptive Treg cells [7]. As human T cells can upregulate Foxp3 transiently upon TCR
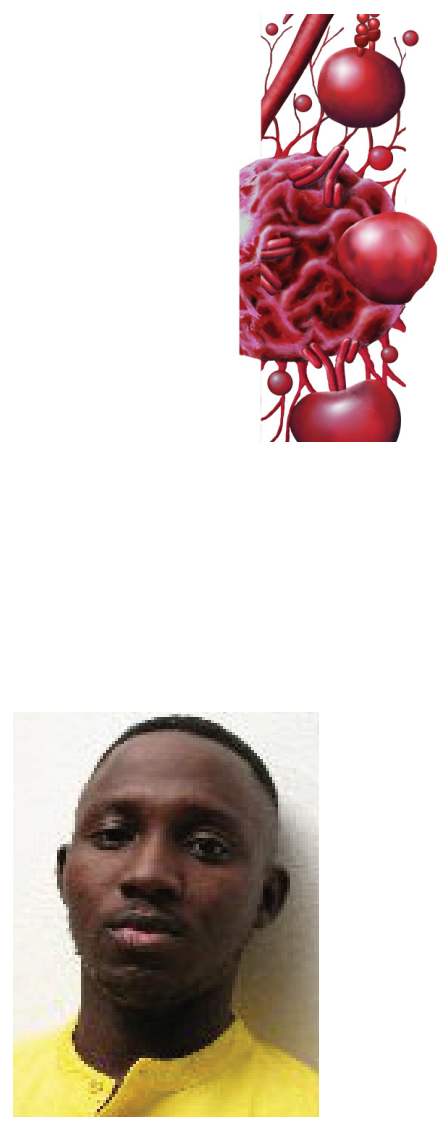

Dennis O Adeegbe Medical Oncology Division, Dana Farber Cancer Institute, Harvard Medical School, 450 Brookline Avenue, Boston, MA 02215, USA

Hiroyoshi Nishikawa Author for correspondence: Division of Cancer Immunology, Exploratory Oncology Research \& Clinical Trial Center, National Cancer Center, Chiba 277-8577, Japan Tel.: +81 471331111 (ext. 91385) Fax: +81471300022

hnishika@east.ncc.go.jp 
activation, it thus remain plausible that a fraction of tumor-infiltrating Foxp3 ${ }^{+} \mathrm{T}$ cells observed in cancer patients is the mixture of Treg cells and Foxp $3^{+}$nonTreg cells. We have previously provided a delineation that can be used to differentiate both naive and activated/effector Treg cells from these foxp3-positive non-Treg-cell fractions [8].

\section{"...Treg cell dampening of antitumor T-cell responses may be orchestrated in series of direct and indirect events involving multiple cell types."}

Although a myriad of soluble factors secreted at the tumor site may shape the phenotype of the Treg cells, it is tempting to speculate that the bulk of Treg cells are antigen-experienced, thus are of an activated/effector phenotype (Figure 1). This is indeed consistent with reports demonstrating the expression of activation markers as well as upregulation of other inhibitory molecules on Treg-cell populations present in the tumor compared with their peripheral counterparts [9,10]. Regardless of their phenotype, one outstanding question is for what purposes are they recruited and how do they achieve their functions? Although there is spare direct evidence, a prevailing dogma is that they are recruited to regulate $T$ cells with a propensity to recognize and respond against tumor-associated antigens. In this context, they essentially impede antitumor T-cell responses. This is not far-fetched given that tumor-related proteins are self antigens, sometimes in altered forms and Treg cells evolved to curb self-reactive $\mathrm{T}$ cells. Once present, tumor-resident Treg cells may exert inhibitory functions against effector $T$ cells in a manner akin to suppression of T-cell proliferation and effector cytokine production seen in vitro [11]. Whether this requires direct contact or is largely mediated by soluble factors derived from the Treg cells is yet to be fully substantiated. Other important modes of suppression by Tregs include inhibition of APC maturation dependent on Treg-cell-CTLA-4 engagement with costimulatory molecules CD80/86 on APC [12]. Furthermore, they may act in tandem with other suppressor types such as myeloid-derived suppressor cells, posing a barrier to efficient infiltration of effector T cells [13]. Thus, Treg cell dampening of antitumor T-cell responses may be orchestrated in series of direct and indirect events involving multiple cell types.

\section{Targeting Treg cells for therapy \\ Depletion}

Various mouse models have explored the depletion of Treg cells in order to demonstrate their contributory role to attenuating T-cell effector function in the tumor microenvironment [2]. Antibodies against $\mathrm{CD} 25$, the high-affinity IL-2R $\alpha$ chain that is constitutively expressed on Treg cells have been used for such purpose although there remains the complication that it could potentially also deplete recently activated, CD25-expressing effector T cells. Similarly in humans, daclizumab, a monoclonal antibody against CD25 and denileukin-Difitox (Ontak), a recombinant protein composed of IL-2 and the active domain of diphtheria toxin, have been utilized for Treg-cell elimination with varying degrees of success [2]. In addition, anti-CCR4 antibody has been demonstrated to show efficacy in selectively eliminating activated/effector subset of Treg cells from cells obtained from cancer patients, and this led to parallel improvement in effector T-cell function when tumor antigen-specific $\mathrm{CD}^{+}$ $\mathrm{T}$ cells were evaluated in vitro [14]. Although depleting strategies are almost always transient, the significant reduction in circulating or tumor-associated Treg-cell frequencies often favors improved antitumor response, exemplified by arrest or decline of tumor growth, improved survival as well as improved effector T-cell function [15]. These observations thus further support the notion that the presence of Treg cells indeed mostly impedes T-cell effector function within tumors. For durable therapeutic response, intermittent but chronic depletion will likely be warranted but this approach especially if achieved via systemic drug administration routes may predispose patients to development of autoimmune diseases in the absence of sufficient numbers of peripheral Treg cells. Thus, careful consideration must be given to such strategies and when possible, local administration or guided delivery into the tumor microenvironment should be favored over systemic treatments.

\section{Functional inactivation}

With the approval of anti-CTLA-4 (ipilimumab) and anti-PD-1 (nivolumab, pembrolizumab) in melanoma patients, there have been increasing efforts toward immunotherapy of cancer with antibodies that target various immune checkpoints. Incidentally, these molecules may have direct or indirect effects on Treg cells [16]. Consistent with this notion, modification in Treg-cell numbers or function have been observed upon anti-CTLA-4 treatment $[17,18]$. Interestingly, its administration potentiates a reshaping of TCR repertoire of peripheral $\mathrm{CD}^{+} \mathrm{T}$ cells toward increased diversity [19], an observation that one might speculatively attribute to its effect on Treg cells, in other words, blocking CTLA-4 inhibitory activity on effector $\mathrm{T}$ cells. While anti-PD-1 antibody administration has not been reported to cause a reduction in Tregcell frequencies, it remains a possibility that it may 


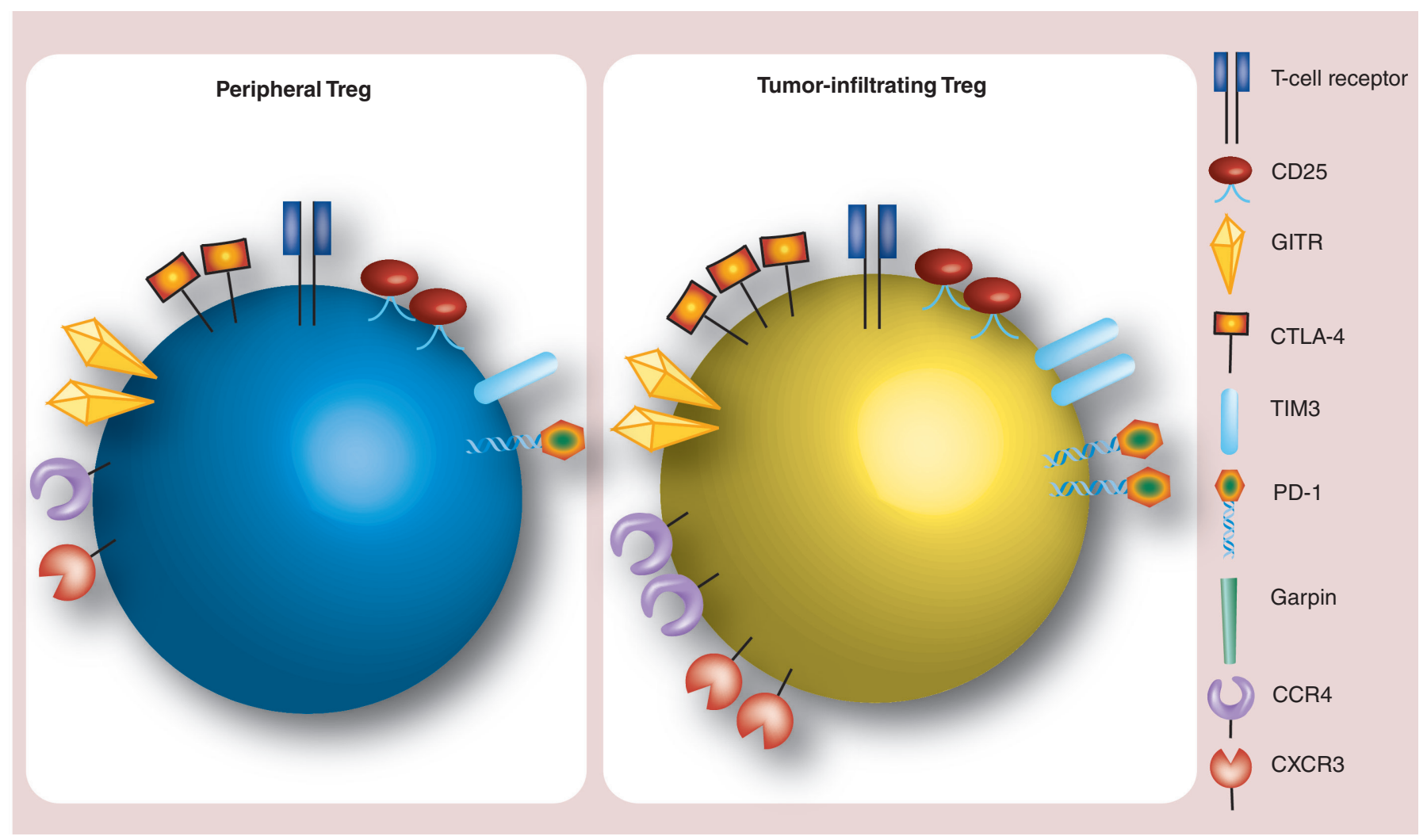

Figure 1. Distinct features of tumor-infiltrating Treg cells as platform for localized control. Relative to their peripheral counterparts, tumor-infiltrating Treg cells upregulate a number of molecular markers some of which are consistent with a more activated phenotype. Molecules such as CD25, GITR which are ubiquitously expressed on Treg cells regardless of their anatomical location offers an avenue for antibody-based depletion. The upregulation of key inhibitory receptor molecules such as TIM-3, PD-1, CTLA-4 or chemokine receptors such as CCR4, CXCR3, as well as activation-induced expression of Garpin on tumor-resident Treg cells, however, could be capitalized upon for their site-specific depletion or functional inactivation. This approach should favor Treg-cell control within the local tumor microenvironment.

have direct effects on Treg-cell function especially in the tumor where its expression is elevated on Treg cells [10]. Other Treg-cell-specific molecules that are of interest include GITR and GARPIN. While the former can be upregulated on activated $\mathrm{T}$ cells, the increased expression of the latter is only confined to Treg cells upon activation and has been suggested to play a role in Treg-cell function [20]. Thus, for therapeutic purposes, attention should be paid to the distinct features exhibited by tumor-infiltrating Treg cells (Figure 1). In this regard, utilization of molecules that may specifically target activated/effector Treg cells for functional inactivation are likely to yield beneficial outcomes since the 'culprit' Treg-cell population within cancers is likely to exhibit such phenotype (Figure 1). An added benefit to this approach is that even if such molecules have Treg-depleting effects, it will likely be mostly confined to those subsets that are antigen-primed and thus, presently participating in the regulation of an ongoing immune activity such as might be expected within the cancer microenvironment.

\section{Perspectives}

While Treg cells have become a formidable T-cell subset with an exaggerated representation in the tumor microenvironment, how to control them without increasing patient's risks to other immune disorders or collateral damage due to off-target toxicity remains a subject of ongoing investigations. Can regulatory $\mathrm{T}$ cells be controlled in cancer? For now, we have resources at our disposal to target them for depletion and functional impairment but the strategies will need to be fine-tuned and optimized preferably for local effect restricted to the tumor and its immediate environment. We are in the age of rapidly emerging new concepts and it remains likely that in the foreseeable future, therapeutic agents that will be able to effectively eliminate subsets of antigen-specific Treg cells at local sites of immune assault, inflammation or disease will become a thing of reality. With such novel agents, controlling Treg cells in cancer settings will likely be a much more surmountable task but until then, the race toward 'Treg-centric' cancer treatment continues and the answer to the topic question remains a half yes. 
Financial \& competing interests disclosure

The authors have no relevant affiliations or financial involvement with any organization or entity with a financial interest in or financial conflict with the subject matter or materials discussed in the manuscript. This includes employ-

\section{References}

1 Sakaguchi S. Naturally arising $\mathrm{CD}^{+}$regulatory $\mathrm{T}$ cells for immunologic self-tolerance and negative control of immune responses. Annu. Rev. Immunol. 22, 531-562 (2004).

2 Nishikawa H, Sakaguchi S. Regulatory T cells in cancer immunotherapy. Curr. Opin. Immunol. 27, 1-7 (2014).

3 Nishikawa H, Sakaguchi S. Regulatory T cells in tumor immunity. Int. J. Cancer 127(4), 759-767 (2010).

4 Facciabene A, Motz GT, Coukos G. T-regulatory cells: key players in tumor immune escape and angiogenesis. Cancer Res. 72(9), 2162-2171 (2012).

5 Chow MT, Luster AD. Chemokines in cancer. Cancer Immunol. Res. 2(12), 1125-1131 (2014).

6 Tan MC, Goedegebuure PS, Belt BA et al. Disruption of CCR5-dependent homing of regulatory $T$ cells inhibits tumor growth in a murine model of pancreatic cancer. J. Immunol. 182(3), 1746-1755 (2009).

7 Adeegbe DO, Nishikawa H. Natural and induced T regulatory cells in cancer. Front. Immunol. 4, 190 (2013).

8 Miyara M, Yoshioka Y, Kitoh A et al. Functional delineation and differentiation dynamics of human $\mathrm{CD}^{+} \mathrm{T}$ cells expressing the Foxp3 transcription factor. Immunity 30 (6), 899-911 (2009).

9 Tanchot C, Terme M, Pere H et al. Tumor-infiltrating regulatory T cells: phenotype, role, mechanism of expansion in situ and clinical significance. Cancer Microenviron. 6(2), 147-157 (2013).

10 Park HJ, Kusnadi A, Lee EJ et al. Tumor-infiltrating regulatory $\mathrm{T}$ cells delineated by upregulation of $\mathrm{PD}-1$ and inhibitory receptors. Cell. Immunol. 278(1-2), 76-83 (2012).

11 Shevach EM. Mechanisms of Foxp $3^{+} \mathrm{T}$ regulatory cellmediated suppression. Immunity 30 (5), 636-645 (2009). ment, consultancies, honoraria, stock ownership or options, expert testimony, grants or patents received or pending, or royalties.

No writing assistance was utilized in the production of this manuscript.

12 Yamaguchi T, Wing JB, Sakaguchi S. Two modes of immune suppression by Foxp $3^{+}$regulatory $\mathrm{T}$ cells under inflammatory or non-inflammatory conditions. Semin. Immunol. 23(6), 424-430 (2011).

13 Lindau D, Gielen P, Kroesen M, Wesseling P, Adema GJ. The immunosuppressive tumour network: myeloid-derived suppressor cells, regulatory $\mathrm{T}$ cells and natural killer $\mathrm{T}$ cells. Immunology 138(2), 105-115 (2013).

14 Sugiyama D, Nishikawa H, Maeda Y et al. Anti-CCR4 mab selectively depletes effector-type Foxp $3^{+} \mathrm{CD} 4{ }^{+}$regulatory T cells, evoking antitumor immune responses in humans. Proc. Natl Acad. Sci. USA 110 (44), 17945-17950 (2013).

15 Oleinika K, Nibbs RJ, Graham GJ, Fraser AR. Suppression, subversion and escape: The role of regulatory $\mathrm{T}$ cells in cancer progression. Clin. Exp. Immunol. 171(1), 36-45 (2013).

16 Page DB, Postow MA, Callahan MK, Allison JP, Wolchok JD. Immune modulation in cancer with antibodies. Annu. Rev. Med. 65, 185-202 (2014)

17 Hodi FS, Butler M, Oble DA et al. Immunologic and clinical effects of antibody blockade of cytotoxic T lymphocyteassociated antigen 4 in previously vaccinated cancer patients. Proc. Natl Acad. Sci. USA 105(8), 3005-3010 (2008).

18 Jie HB, Schuler PJ, Lee SC et al. CTLA- $4^{+}$regulatory T cells increased in cetuximab treated head and neck cancer patients suppress NK cell cytotoxicity and correlate with poor prognosis. Cancer Res. 75(11), 2200-2210 (2015).

19 Cha E, Klinger M, Hou Y et al. Improved survival with $\mathrm{T}$ cell clonotype stability after anti-CTLA- 4 treatment in cancer patients. Sci. Transl. Med. 6(238), 238 ra270 (2014).

20 Wang R, Kozhaya L, Mercer F, Khaitan A, Fujii H, Unutmaz D. Expression of GARP selectively identifies activated human Foxp $3^{+}$regulatory T cells. Proc. Natl Acad. Sci. USA 106(32), 13439-13444 (2009). 